\title{
Pratiques
}

Linguistique, littérature, didactique

145-146 | 2010

Didactique du français (1)

\section{Recherche et didactique}

\section{Caroline Masseron}

\section{OpenEdition}

Journals

Édition électronique

URL : http://journals.openedition.org/pratiques/1487

DOI : 10.4000 /pratiques. 1487

ISSN : 2425-2042

\section{Éditeur}

Centre de recherche sur les médiations (CREM)

\section{Édition imprimée}

Date de publication : 15 juin 2010

Pagination : 8-11

\section{Référence électronique}

Caroline Masseron, «Recherche et didactique », Pratiques [En ligne], 145-146 | 2010, mis en ligne le 15 juin 2010, consulté le 14 novembre 2019. URL : http://journals.openedition.org/pratiques/1487 ; DOI : $10.4000 /$ pratiques. 1487

(C) Tous droits réservés 


\section{Recherche et didactique Table ronde}

Le 7 novembre 2009, à l'Université de Metz, s'est tenue une table ronde sur les rapports entretenus entre la recherche, la formation et la didactique (du français). Y ont participé respectivement Claudine Garcia-Debanc, Elisabeth Nonnon, Elisabeth Bautier, Bernard Combettes, Claire Margolinas et Raymond Michel ${ }^{(1)}$. L'événement en célébrait un autre : le départ à la retraite de Marceline Laparra, grande figure s'il en fût de la didactique du français, qu'il s'agisse des enjeux scolaires de formation initiale et continue ou des batailles politiques menées pour les publics les plus fragiles (FLE, illettrisme, ZEP); il convenait d'adresser à Marceline le message solennel et collectif de notre reconnaissance.

Les pages qui suivent témoignent de ce message. Leur lecture renforce cette impression, déjà très sensible sur le moment, de qualité des échos que chacun a su donner à la voix singulière qu'il a fait entendre. En effet, les intervenants invités, au-delà des domaines, des problématiques et des institutions qu'ils incarnent, ont $\mathrm{su}$, avec toute la finesse d'analyse, l'esprit critique et la culture professionnelle qu'on leur connaît, évoquer les notions et questions impliquées par la thématique, pourtant vague, à laquelle ils se sont soumis amicalement ; c’était une gageure, étant donné le peu de temps imparti à chacun et la complexité « politique » et épistémologique des problèmes soulevés.

L'ouverture de Claudine Garcia-Debanc donne le cadre introductif nécessaire pour engager une discussion sur les fondements de la didactique du français, envisagée dans sa double appartenance à la formation et à la recherche. Reprenant les trois paliers de la transposition didactique (les savoirs théoriques, les savoirs enseignés et les savoirs appris), l'auteure complète cette approche à l'aide des notions de «configuration » disciplinaire (J.-F. Halté) et de «modèle disciplinaire en

(1) Jean-Marie Privat a amicalement accepté d'orchestrer les débats. Il 1'a fait avec un art consommé du «spectacle culturel», dont il ne reste hélas que quelques bribes sur la copie vidéo. 
actes »(C. Garcia-Debanc). Cet éventail notionnel lui permet de caractériser les enjeux scolaires et sociaux de la discipline (préconisations, certifications et pratiques langagières dans la classe et en dehors), et d'apprécier les écarts entre des discours d'intention et des pratiques effectives. L'ancrage institutionnel et international de la didactique du français est donc rappelé avec d'autant plus de vigueur que la situation française - qu'il s'agisse des programmes ou des instances de formation - s'est affaiblie dans la période récente, ce qui a eu sans doute des conséquences sur la conception praxéologique de transformation démocratique du système. En revanche, les liens, organiques et nécessaires, avec les recherches disciplinaires - en sciences du langage, sciences de l'éducation, et littérature sont moins clairs, sauf à dire que les méthodes et résultats obtenus doivent être irréprochables et que « le grain d'analyse du traitement» y est variable. À vrai dire, l'équilibre est sans doute impossible à trouver sans une collaboration de travail, institutionnelle, scientifique et pratique, à long terme, entre les différents agents de la noosphère, y compris bien sûr les enseignants eux-mêmes. La situation politique actuelle divise les agents, au point que chacun a pu momentanément intériorisé l'idée qu'il «s'en sortirait » mieux tout seul, ou au sein d'une coterie scientifique de petite dimension. Dans son exposé de cadrage, Claudine Garcia-Debanc sous-entend ces obstacles politiques qui sont de premier ordre, même si les circonstances lui interdisent probablement un registre trop pessimiste.

Elisabeth Nonnon prolonge la réflexion amorcée sur les liens entre institutions et terrains d'exercice professionnel, en revenant sur le dispositif local qu'il lui a fallu concevoir, et en retraçant les problèmes épistémologiques et méthodologiques - pratiques - auxquels elle s'est trouvée confrontée. Les questions soulevées sont innombrables et passionnantes, même si elles communiquent parfois une sorte de vertige à imaginer ce qui reste à entreprendre. Au rang des tâches urgentes, figure la nécessité de dresser un bilan sans complaisance des recherches en didactique du français et des résultats qu'elles ont donnés. En effet, compte tenu de sa dimension axiologique (modélisante), de ses acteurs impliqués et des conditions pratiques de son effectuation (enquête de terrain et recueil de données), la recherche en didactique du français est soumise à des aléas et à des contradictions, qui sont pour une part issus de l'histoire de la discipline et du champ. Elisabeth Nonnon énumère, avec une responsabilité critique dûment mesurée, quelques indicateurs de faiblesse qui devraient alerter la communauté : la fragilité, en termes de résultats, des études de cas, le déséquilibre des objets investis dans le catalogue des recherches en didactique du français, l'absence de propositions en matière de progression en langue, la discrétion de certains domaines comme le vocabulaire, l'insuffisance des retours critiques sur ce qu'a produit la didactique du français, etc. L'évaluation des " coûts »symboliques et professionnels (insécurité du maître, temps pris dans la classe, hypothèses et résultats des recherches engagées en termes d'apprentissages) est au cœur de la réflexion à laquelle nous conduit E. Nonnon.

Bernard Combettes, quant à lui, livre ses réflexions sur la didactique de la langue. Il le fait à partir d'une synthèse sur la situation présente et les malentendus persistants qui caractérisent 1'enseignement « grammatical » (langue et grammaire, grammaire intuitive et grammaire explicite, oral et écrit, normes et variations, texte et phrase, etc.). Au-delà de ces premières considérations critiques, B. Combettes suggère que l'on modifie les contenus de l' " enseignement grammatical », d'une part en y adaptant les objets d'analyse et les paliers de traitement aux objectifs visés ; et d'autre part, en y intégrant les apports novateurs des grammai- 
res fonctionnelles (Givon, Dik) ou les développements sur les variations langagières, envisagées selon un continuum (au lieu du jugement normatif simplificateur), dans leur dimension diachronique (le «changement linguistique ») et leur perspective sociolinguistique (les variétés du français).

Raymond Michel, pour sa part, traite des «études littéraires » et s'insurge - à l'instar d'Yves Citton ${ }^{(2)}$ dont il partage le plaidoyer en faveur de lectures dites « actualisantes »-contre la délégitimation dont elles sont l'objet. L'enjeu est de retrouver l'espace de liberté du lecteur dont l'activité (l'expérience, langagière, cognitive et sensible, des fictions) doit être pensée à part entière dans « l'interlocution littéraire », où l'interprétation de la fiction relève d'un double mouvement d'individuation symbolique et de socialisation intelligente. L'adhésion aux valeurs d'autonomie de la pensée critique et d'altérité fondatrice, si nous suivons bien R. Michel, pose les jalons d'une discussion nécessaire sur les abus formalistes des «genres-types » et le retour à des modalités d'itinéraire interprétatif, qui seraient plus novatrices, singulières, engageantes et libres.

Claire Margolinas, didacticienne des mathématiques, élargit le propos en inscrivant son intervention dans un champ pluridisciplinaire, au sens où elle permet de tisser des trames notionnelles d'une didactique à une autre, en l'occurrence des mathématiques au français. Rappelons que C. Margolinas collabore au groupe de recherche sur les inégalités scolaires (le groupe RESEIDA, dirigé par E. Bautier et J.-Y. Rochex), et que ses travaux de recherche sont nourris par des concepts et des auteurs qui ne sont pas tout à fait inconnus en didactique du français. Jean-François Halté, à Pratiques, avait contribué à les faire connaître : Chevallard et Brousseau, par exemple, l'un pour le concept de transposition, l'autre pour ceux de $\mathrm{si}^{-}$ tuation, de milieu et de structuration. À l'instar des articles que l'auteure cosigne avec Marceline Laparra, sa contribution à la table ronde a l'immense intérêt de montrer comment s'opère ce passage d'une situation ordinaire (« compter des jetons », en grande section de maternelle) à son analyse épistémologique. Comment s'apprend - ou ne s'apprend pas - le concept d'énumération ? Mais, auparavant, qu'appelle-t-on " énumérer " ? dénombrer des points, "désigner des objets une fois et une seule jusqu'à ce qu'il n'y en ait plus », "réciter la comptine » (un, deux, trois, quatre...) ? Les opérations et procédures qui sont impliquées sont ici nommées, définies, illustrées et, pour notre plus grand profit, décomposent une activité qu'il serait tentant faute de ces éclairages de juger « simple » et « naturelle ». Claire Margolinas rapporte l'observation passionnante d'un cas (Angélique, en GS de maternelle) où la réussite de l'activité (dans le résultat final) révèle en réalité une stratégie de contournement de la tâche, qui esquive ainsi l'apprentissage impliqué (tri des étiquettes : énumération).

Le point de vue d'Elisabeth Bautier est d'autant plus précieux que, transfuge des sciences du langage, l'appartenance actuelle de son auteure aux sciences de l'éducation, la met dans une position privilégiée pour appréhender les problématiques de recherche qui interrogent l'enseignement du français dans ses relations avec les apprentissages fondamentaux et les inégalités scolaires. Les résultats de son investigation sont formulés comme autant de paradoxes qu'il convient de garder en mémoire. Le premier de ces paradoxes concerne les enseignables et la priorité accordée ces dernières années à l'énonciation et aux types de discours, sans

(2) Lire, interpréter, actualiser. Pourquoi les études littéraires? Paris, Éditions Amsterdam, 2007. 
que pour autant se réalisent les progrès que l'on pouvait en attendre du côté des écrits de savoir et de la variété des genres scolaires. Un second paradoxe a trait à l'inflation de travaux sur les interactions qui, rapportée à l'affaiblissement du « discours instructeur » (Bernstein), a renforcé, au lieu de discuter, une conception transparente du médium langagier. Enfin, citons un dernier paradoxe : celui qui consiste à prôner le socioconstructivisme comme théorie de référence, sans prendre la précaution d'étayer les objets de la psycholinguistique (les opérations cognitives du sujet en formation) par ceux de la sociologie (les inégalités langagières, sociales et scolaires). E. Bautier souhaite pour sa part que les dimensions contextuelles et sociales des situations d'enseignement constituent un axe de recherche, dont l'importance ne soit plus minorée. Elle souligne par ailleurs la dimension temporelle d'études qui pourraient être «longitudinales » et qui, si on les compare aux études de cas plus ponctuelles (aujourd'hui dominantes dans la didactique du français), pourraient apporter leur lot de connaissances nouvelles sur les facteurs d'obstacle à l'apprentissage et le tri entre des phénomènes distincts (développement du sujet, forme scolaire de l'apprentissage, contexte de l'enseignement dispensé). Enfin, un des effets non négligeables du travail sur la temporalité des apprentissages serait de mieux saisir ce qui est invisible dans l'instant d'une observation, ou ce qui est rendu moins visible car non enseigné. Entreraient dans cette catégorie des objets invisibles le dispositif matériel d'une situation d'enseignement et la sophistication des relations entre littératie et oralité.

En conclusion, Pratiques peut s'enorgueillir de la réalisation de la table ronde qu'on va lire, et les participants remerciés d'avoir œuvré à sa qualité. La réflexion collective, savante, responsable, qui en est issue n'a fait appel à aucune technologie de pointe mais à ce qui est, aujourd'hui, plus rare peut-être : une forme de conscience politique, ou de culture commune, qui soit, par-delà des intérêts institutionnels divergents, pluridisciplinaire et attentive aux enjeux socio-scolaires des démarches de recherche et de formation.

Et encore une fois, merci à Marceline Laparra d'avoir inspiré le dispositif et l'échange de vues qui en est advenu.

Caroline Masseron

NB : les auteurs n'ont pas adopté tout à fait les mêmes conventions (bibliographie, appel de notes, sous-titres, etc.) quand ils ont réécrit leur intervention orale. Il ne nous est pas paru qu'il faille coûte que coûte « harmoniser» les contributions. 